\title{
REVIEW
}

\section{Alcohol-related dementia: an update of the evidence}

\author{
Nicole J Ridley ${ }^{1,2}$, Brian Draper ${ }^{* 1,3}$ and Adrienne Withall ${ }^{4}$
}

\begin{abstract}
The characteristics of dementia relating to excessive alcohol use have received increased research interest in recent times. In this paper, the neuropathology, nosology, epidemiology, clinical features, and neuropsychology of alcoholrelated dementia (ARD) and alcohol-induced persisting amnestic syndrome (Wernicke-Korsakoff syndrome, or WKS) are reviewed. Neuropathological and imaging studies suggest that excessive and prolonged use of alcohol may lead to structural and functional damage that is permanent in nature; however, there is debate about the relative contributions of the direct toxic effect of alcohol (neurotoxicity hypothesis), and the impact of thiamine deficiency, to lasting damage. Investigation of alcohol-related cognitive impairment has been further complicated by differing definitions of patterns of alcohol use and associated lifestyle factors related to the abuse of alcohol. Present diagnostic systems identify two main syndromes of alcohol-related cognitive impairment: ARD and WKS. However, 'alcoholrelated brain damage' is increasingly used as an umbrella term to encompass the heterogeneity of these disorders. It is unclear what level of drinking may pose a risk for the development of brain damage or, in fact, whether lower levels of alcohol may protect against other forms of dementia. Epidemiological studies suggest that individuals with ARD typically have a younger age of onset than those with other forms of dementia, are more likely to be male, and often are socially isolated. The cognitive profile of ARD appears to involve both cortical and subcortical pathology, and deficits are most frequently observed on tasks of visuospatial function as well as memory and higher-order (executive) tasks. The WKS appears more heterogeneous in nature than originally documented, and deficits on executive tasks commonly are reported in conjunction with characteristic memory deficits. Individuals with alcohol-related disorders have the potential to at least partially recover - both structurally and functionally - if abstinence is maintained. In this review, considerations in a clinical setting and recommendations for diagnosis and management are discussed.
\end{abstract}

It is well established that excessive and prolonged alcohol use can lead to permanent damage to the structure and function of the brain [1]. Despite this, there is little consensus on the characteristics of a dementia syndrome related to sustained alcohol abuse or its relationship to Wernicke-Korsakoff syndrome (WKS). After a long period of neglect, research interest has increased in recent years and has been spurred on by clinical demand, increased reported rates of alcohol abuse in older people, and increasing alcohol consumption by women $[2,3]$. In this paper, we aim to review the neuropathology, nosology, epidemiology, clinical features, and neuropsychology of alcohol-related dementia (ARD) and WKS. To

\footnotetext{
*Correspondence: b.draper@unsw.edu.au

'Dementia Collaborative Research Centre, School of Psychiatry, University of New South Wales, Anzac Parade, Kensington, NSW, 2052, Australia
}

Full list of author information is available at the end of the article retrieve papers for the purpose of this review, the search terms (alcohol OR alcoholism) AND (dementia OR brain damage OR brain injury OR cognitive impairment) were used as keywords in the Medline and PsycINFO databases. Additional terms included Wernicke's encephalopathy, Korsakoff, and Alcohol Amnestic Disorder. Reference lists were also scanned for relevant papers.

\section{The controversy of alcohol-related dementia}

Present diagnostic criteria for alcohol-associated cognitive disorders focus on two main syndromes of impairment: WKS and ARD. ARD has enjoyed little recognition as a discrete clinical entity and this is due mainly to a lack of a distinct pathophysiological profile [4,5]. Much of the debate surrounding ARD encompasses whether it is possible to have a dementia that is the direct result of ethanol neurotoxicity - a primary alcoholic dementia or whether the clinical presentation of dementia represents another underlying pathology (that is, thiamine deficiency) or multiple factors (for example, neurotoxicity 
in combination with nutritional deficiencies). Attempts to clarify this have been hindered by confounding factors that often accompany the lifestyles of alcohol abusers, such as head injury, psychiatric and other substance abuse co-morbidities, and a higher rate of vascular risk factors [6]. In some countries, terms such as 'alcoholrelated brain damage' or 'alcohol-related brain injury' are preferred over ARD to reflect the heterogeneity of alcohol-related cognitive disorders in both etiology and clinical presentation [7].

\section{The pathophysiology of alcohol-related dementia}

Autopsy evaluations suggest that up to $78 \%$ of individuals with diagnosed alcoholism demonstrate some degree of brain pathology [8]. Neuroimaging and neuropathological evidence show prominent white matter loss (most notable in the prefrontal cortex, corpus callosum, and cerebellum) and neuronal loss in the superior frontal association cortex, hypothalamus, and cerebellum [1,9]. The frontal lobes of individuals with diagnosed alcoholism appear particularly susceptible to damage, with evidence of markedly decreased neuron density, volume shrinkage, and altered glucose metabolism and perfusion [10]. Partial recovery of white matter disturbances can occur with abstinence, and magnetic resonance imaging studies indicate early reversibility of white matter shrinkage that is accompanied by clinical improvement in cognitive and motor abilities $[11,12]$. The mechanism behind recovery from white matter damage is thought to involve the restoration of myelination and axonal integrity, but is vulnerable to repeated disruption if drinking is resumed [11]. Harper [1] concludes that the brain pathology of abusers of alcohol likely has two components: one of permanent change, the other transient.

While these findings reflect general structural changes in chronic abusers of alcohol, what lesions characterize a clinically identifiable dementia and the neuropathological process that underlies this process remain in dispute. The 'neurotoxicity' hypothesis suggests that the direct physiological effects of chronic alcohol exposure can cause neuronal loss through glutamate excitotoxicity, oxidative stress, and the disruption of neurogenesis [13]. In particular, drinking patterns of repeated binges and withdrawal periods may enhance neuronal injury through increased vulnerability of upregulated N-methyl-Dasparate (NDMA) receptors to glutamate-induced excitotoxicity. Support for the neurotoxicity hypothesis emerges from animal studies, which have demonstrated doserelated ethanol-induced damage to brain structures including the hippocampus, hypothalamus, and cerebellum - that correspond with impairments in memory and learning $[14,15]$. Cholinergic neurotransmission in the basal forebrain, which plays a key role in attention, learning, and memory, also appears to be impacted by prolonged intake of alcohol. Imaging studies of 'uncomplicated alcoholics' - individuals with no history of nutritional deficiency, hepatic failure, or other indirect forms of brain injury - confirm structural abnormalities, including changes to the corpus callosum, pons, and cerebellum [12]. However, the permanence of such changes, and whether they relate to neurotoxicity in isolation, remains to be established [1].

Another hypothesis is that thiamine (vitamin $\mathrm{B}_{1}$ ) deficiency is primarily responsible for the development of ARD. Individuals with alcohol use disorders are at particularly high risk of thiamine deficiency, not only from poor dietary nutrition but because alcohol directly compromises thiamine metabolism [16]. Thiamine deficiency can lead to Wernicke's encephalopathy (WE), an acute neurological disorder characterized by the clinical triad of oculomotor abnormalities, cerebellar dysfunction, and altered mental state and by the traditionally defined pathology of neuronal loss and hemorrhagic lesions in the paraventricular and periaqueductal grey matter. Not all individuals with WE show the triad of neurological symptoms, and the severity of signs is likely related to the extent of the underlying pathology [17]. To increase diagnostic accuracy of WE, refined operational criteria specify a minimum of two symptoms for diagnosis, a guideline recently endorsed by the European Federation of Neurological Societies (EFNS) [18,19]. Long-term outcomes of WE can include development of a syndrome of profound memory impairment - Korsakoff syndrome (KS) - that appears to be related to additional disruption to diencephalic and hippocampal circuitry. As KS shares similar pathological substrates and often follows an episode of WE, it is commonly referred to as the Wernicke-Korsakoff syndrome [1]. Increasing evidence suggests that the WKS encompasses a spectrum of pathological, neurological, and cognitive impairments resulting from thiamine deficiency [4]. The heterogeneity in presentation of the WKS, in combination with a lack of distinct pathological evidence for ARD, has led to the suggestion that cases of ARD are variants of the WKS [20]. Other evidence suggests that ARD and WKS are distinct disorders with overlapping clinical symptoms and associations such as peripheral neuropathology and ataxia [21].

\section{The relationship of Wernicke-Korsakoff syndrome to alcohol-related dementia: pathophysiology}

In cases found to have WKS pathology at autopsy, the syndrome was correctly diagnosed in only around $20 \%$ of individuals prior to death [22]. Overlap with ARD has been noted, and it has been hypothesized that inactive (chronic) WE is likely to be the main underlying pathology in both KS and ARD [23,24]. Individuals with diagnosed KS and ARD show greater loss of neurons in 
the nucleus basalis than individuals with uncomplicated alcoholism, although this needs replication $[25,26]$. Graded deficits in regional brain volumes, in which 'uncomplicated alcoholics' demonstrate similar but less severe lesions than individuals with WKS (mammillary bodies, thalamus, cerebellar hemispheres, and vermis), also suggest that subclinical bouts of thiamine deficiency contribute to mild structural changes in alcohol-related disorders [17]. In individuals with diagnosed KS, reports of abnormalities in brain regions beyond those associated with thiamine depletion also provide support for a 'spectrum' of impairment in WKS that likely relates to damage from both thiamine and direct neurotoxicity or a compound effect of both [17].

Preliminary reports from animal models suggest that thiamine deficiency and direct alcohol neurotoxicity produce similar brain effects. These include loss of cells in the basal forebrain, hippocampal acetylcholine hypofunction, and shrinkage of frontal grey and white matter, with thiamine deficiency characterized by additional lesions in the diencephalon [27]. Vetreno and colleagues [27] suggested that the interaction between ethanol and thiamine deficiency does not produce more behavioral or neural pathology, with the exception of reduced white matter, than long-term thiamine deficiency alone; however, synergic effects have been noted elsewhere [28]. Notably, pure cases of thiamine deficiency, unaccompanied by chronic and excessive alcohol consumption (such as in cases of malnutrition), show a low rate of progression to KS [29], giving credence to the idea that an interaction of causative factors is responsible for the lasting cognitive deficits seen in alcohol-related disorders.

\section{How much is too much?}

The relationship between the amount of alcohol use and cognitive outcomes is complicated by differing definitions of drinking levels in the literature, and this complication relates in part to the varying definitions of a 'standard drink' from country to country. For example, a standard drink in the United Kingdom contains a relatively low 8 grams of alcohol, compared with 10 grams in Australia, 14 grams in the US, and 19.75 grams in Japan [30].

'High' levels of alcohol consumption can range from 10 'standard' drinks a week [31] to more than 9 'standard' drinks a day [32]. Reduced frontal lobe volume has been associated with an amount of 418 grams a week but has not correlated with lower levels of consumption [14]. One review suggested that consumption of five to six drinks per day (which, by US standards, equates to 70 to 84 grams) over extended periods results in 'cognitive inefficiencies', while consumption of 10 or more standard drinks a day manifests as moderate cognitive deficits equivalent to that found in individuals with diagnosed alcoholism [33].
The differing elements of drinking patterns (for example, duration and severity of abuse, binge, and withdrawal periods) as well as difficulties gaining an accurate selfreport of past drinking have further complicated attempts to link drinking levels to later cognitive impairment. Estimates of past drinking habits of individuals diagnosed with ARD have included up to 60 years of drinking (and up to 120 drinks a week at heaviest), although there is significant variability in length and severity of drinking [34]. Oslin and colleagues [35] suggested that a five-year history of consuming 35 standard drinks a week for men and 28 for women constitutes a sufficient level of neurotoxic burden to risk the development of ARD, but this needs verification.

Alcohol has been examined as a risk factor for other dementia syndromes. There are suggestions of a U- or J-shaped relationship between alcohol consumption and dementia, with low-moderate drinking levels reducing the risk of overall dementia but heavy use increasing the risk [14]. Low to moderate alcohol use is thought to reduce the risk of coronary artery disease and ischemic stroke through the inhibitory effect of ethanol on platelet aggregation and reduction of inflammatory markers and by alteration of the serum lipid profile [36]. The antioxidant effect of polyphenols or ethanol itself might also provide neuroprotection [14]. Alternatively, heavier drinking may contribute to adverse cerebrovascular changes (hypertension and raised triglycerides) and increased risk of arterial thrombosis, cardiac disorders, and strokes [6]. A meta-analysis examining the association of ethanol and incident dementia concluded that small amounts of alcohol likely protects against Alzheimer's disease but not against vascular dementia [37]. However, others suggest that the benefit of moderate drinking applies to all forms of dementia [38]. This uncertainty was emphasized in a recent 20-year study ( $\mathrm{n}=1,300$ women at least 65 years old), which reported that moderate alcohol consumption was not protective against dementia. Furthermore, women who increased their alcohol consumption over the course of the study had an increased risk of developing dementia [39]. Interestingly, animal models have shown that low concentrations of alcohol protect cultured cortical and hippocampal neurons against the synapse damage induced by amyloid- $\beta$ and $\alpha$-synuclein, providing a pathological explanation for reports that alcohol consumption protects against the development of specific dementia syndromes [40].

\section{Nosology?}

The current DSM-IV (Diagnostic and Statistical Manual of Mental Disorders, 4th edition) criteria for 'alcoholinduced persisting dementia' specify the persistence of cognitive and functional decline following cessation of alcohol consumption, with all other causes of dementia 
excluded [41]. Oslin and colleagues [35] attempted to improve the validity and reliability of ARD diagnosis by standardizing alcohol consumption criteria for a 'probable' diagnosis of ARD (length and severity of alcohol use) and specifying a minimum abstinence time for a dementia diagnosis to be considered. These guidelines were not meant to be definitive and were designed with the intention of stimulating further research. Even so, further use of their criteria has been limited to a handful of studies [42-44] that have inconsistently adopted elements of the criteria (for example, some have excluded participants with previous acute symptomatology of WKS) and differed in participants' ages, education level, and global cognitive function. Thus, current diagnostic criteria for ARD have been inadequately tested.

WKS is classified in the DSM-IV as 'alcohol-induced persisting amnestic disorder, and memory disturbance is the key diagnostic feature [41]. International Statistical Classification of Diseases and Related Health Problems (ICD-10) criteria for 'alcohol-induced amnesic syndrome' [45] are more descriptive and detail impairment of recent memory and disturbance of time sense in the absence of impaired immediate recall or generalized cognitive impairment.

Another term that is becoming widely used is 'alcoholrelated brain damage' (ARBD) (also called alcohol-related brain impairment), which in some countries has superseded the use of DSM-IV nosology [7]. Some authors do not encompass specific neurological disorders that present with a distinct clinical and neuropathological presentation (for example, Marchiafava-Bignami disease) within this terminology [20], whereas others group these under the ARBD banner [7]. For many clinicians, this term is preferential to ARD because it better accounts for the heterogeneity of presentations, avoids the stigma associated with the term dementia, and distinguishes the non-progressive nature of ARD from other degenerative disorders. DSM-5 looks likely to adopt a similar inclusive approach, with plans to have categories of major and minor 'neurocognitive disorder due to substance disorder', which are likely to include an 'amnesticconfabulatory (Korsakoff)' subtype, although this is yet to be finalized [46].

\section{Prevalence of alcohol-related cognitive disorders}

Given the lack of operationally defined diagnostic criteria, it is not surprising that incidence and prevalence estimates of ARD vary in the literature. Epidemiological findings generally have been derived from population studies that relate patterns of alcohol consumption and dementia. A review by Ritchie and Villebrun [47] established that studies have indicated a high prevalence of alcohol abuse in patients with dementia (9\% to $22 \%$ ) and high rates of dementia in alcohol abusers (10\% to
$24 \%)$, although most studies did not specify the type of dementia. The range in rates may be explained at least partially by differences in criteria for 'heavy' alcohol use, varying age limits, and differences in sampling. ARD cases generally have a younger age of onset, and consequently studies that exclude those under 60 years of age may miss a significant proportion of cases [47]. Prevalence studies of dementia subtypes in nursing homes have reported ARD to account for $10 \%$ to $24 \%$ of all dementias $[43,47,48]$, which is likely higher than in the general population. Rates of ARD in dementia cases identified in neurology and memory clinics tend to be lower (around 3\% to 5\% $[49,50]$ ), which may indicate the lack of referrals of these patients to such clinics. Rates of ARD of around 10\% were found in an English epidemiological study of younger-onset dementia in specific London districts (onset of less than 65 years) [51]. The effect of age was demonstrated in an Australian analysis of hospital admissions of more than 20,000 dementia patients who were at least 50 years old: ARD was found in $1.4 \%$ of all patients with diagnosed dementia but in $22 \%$ of dementia patients under 65 [52].

Individuals with ARD are often male, have co-morbid mental and physical conditions (including liver and digestive diseases), and are likely to be identified through hospital admissions $[43,52]$. Social isolation appears to be a significant factor in the poor identification and treatment of ARD, and a high proportion of patients are unmarried or do not have the support of family or friends $[43,53]$. These gender and social findings are consistent with reported characteristics of individuals who are heavy users of alcohol [2]. There has been little examination of the prevalence of co-morbid substance abuse, head injuries, or psychological co-morbidities in the ARD population despite evidence that these are linked to the presence and maintenance of substance use disorders in both younger and older adults [13,54].

Most cases of WKS in developed countries relate to the misuse of alcohol, although WKS syndromes following gastrointestinal disorders and systemic diseases can also contribute. While there is no direct correlation between the prevalence of WE and per capita consumption of alcohol, the introduction of thiamine supplementation programs in some countries, as well as general dietary habits, also influences overall rates [16]. Prevalence rates of WKS identified post-mortem are thought to be between $1 \%$ and $2 \%$ of the general population and around $10 \%$ of alcohol misusers in Western countries $[16,19]$. A study of KS in The Netherlands reported a prevalence of 48 per 100,000 inhabitants [55], and incidence rates of KS in the East End of Glasgow, Scotland, were estimated at around 8 per 100,000 in 1995, a seven-fold increase from 1990 [56]. A study of hospital admissions of patients at least 50 years old identified 126 cases of KS $(0.05 \%$ of all 
admissions) and 77 cases of WE (0.03\% of admissions), although there was some overlap in diagnostic groups [52]. There is a need for further epidemiological study of this population by using standardized criteria for diagnosis to increase accuracy in identification of underlying WKS neuropathology and allow overall prevalence rates to be established.

\section{Neuropsychological findings}

Alcohol can have acute and chronic effects on cognitive function. Direct intoxication impairs most cognitive skills and in excess may lead to stupor and respiratory depression. Acute withdrawal in long-term alcohol abusers can result in tremor, hallucinations, seizures, agitation, and fluctuating levels of alertness [14].

Cognitive and behavioral changes specific to ARD have received limited investigation. Unlike patients with other dementia syndromes, patients with alcohol-induced dementia are unlikely to demonstrate language impairment, an observation incorporated into the ARD criteria proposed by Oslin and colleagues [21,35]; however, preserved confrontational naming has not been shown in all studies [44]. In comparison with individuals with Alzheimer's disease, ARD groups generally performed better on semantic tasks (confrontational naming, category fluency, and general knowledge) and on verbal memory recognition measures despite generally equivalent verbal learning and overall delayed recall to Alzheimer's disease groups [34,44,57]. However, the ARD groups had poorer performance on visuospatial measures, including clock drawing and copying tasks. Deficits on tasks of working memory [44], motor speed [34], and executive function (verbal abstract reasoning [44] and letter fluency [34]) have also been observed in ARD samples in comparison with healthy controls. While findings are limited by small samples (which in some cases overlapped between studies) as well as differences in group characteristics (that is, global dementia severity), Munro and colleagues [57] proposed that the clinical profile of ARD reflects both cortical and subcortical pathology. This was supported by a recent SPECT (single-photon emission computed tomography) study that reported reduced regional cerebral blood flow in the frontal cortices, basal ganglia, and thalami of patients with ARD [42].

The neuropsychology of WKS has been the subject of more extensive investigation. Patients typically demonstrate profound anterograde amnesia and impaired recall of past events, with a temporally graded deficit in which recall is better for more remote time periods [58]. Implicit memory and procedural memory are comparatively spared. Other cognitive functions apart from memory may be disturbed, and impaired executive functions, visuoperceptual difficulties, and disturbed working memory have been observed [59]. Executive deficits have been identified in $80 \%$ of patients with KS [60]. Difficulties are most frequently detected on tasks assessing higher-order organization, planning, and cognitive flexibility (for example, verbal fluency and divided attention) $[60,61]$. There is also evidence for variable intellectual function in WKS [62]. In a review of evidence for variability in WKS, Bowden [4] remarks that empirical evidence suggests that the chronic phase of WKS is more accurately described as 'dementia-like deterioration' rather than severe and selective amnesia.

Abstinence for as little as a week typically resolves many of the deficits associated with heavy alcohol consumption, and further recovery of cognitive abilities can continue over several years. The pattern and rate of cognitive recovery are not yet fully understood; however, there is some suggestion that verbal deficits resolve faster than visuospatial difficulties [13]. Executive function, working memory, and perceptual and motor impairments commonly endure following short-term abstinence, which has been proposed to partly reflect compromised frontocortico-cerebellar functional networks [10]. Recovery of cognitive skills appears to be linked to amount of recent alcohol use and duration of abstinence rather than lifetime alcohol consumption. Multiple withdrawals and binge drinking may significantly exacerbate cognitive deficits $[32,63]$. Older drinkers show greater alcoholrelated cognitive changes and are less likely to recover function once they cease drinking, even after drinking history is controlled for [13]. Other factors such as gender and education also likely contribute to vulnerability to cognitive impairment, and lower levels of education are associated with less recovery over time and females appear to be more vulnerable to cognitive impairment despite generally lower drinking levels than men $[13,63]$. It is unclear whether education is a protective factor or whether low pre-morbid intelligence is a risk for both cognitive impairment and poor educational attainment [13]. It has also been suggested that genetic influences - such as a family history of alcoholism - may be a risk factor for development of alcohol use cognitive disorders, with cognitive dysfunction potentially predating alcohol misuse [13].

Another feature common to ARD and WKS is stability, and even improvement, in cognitive functioning over time provided that abstinence is maintained. A two-year follow-up of nursing home residents with dementia reported that patients with ARD demonstrated a stabilization of cognitive and functional status, as opposed to individuals with Alzheimer's disease or vascular dementia who experienced a general decline [43]. This is consistent with reports from a Scottish study [64] of stability and even improvement in some cognitive domains for an ARD group over a one-year follow-up. Similarly, 
individuals with WKS also have potential for recovery, although this is not commonly recognized [4]. Improvements in general knowledge, visual long-term memory, and verbal fluency have been found in abstinent individuals with $\mathrm{KS}$ over two years, and cognitive improvement is associated with higher pre-morbid education and fewer detoxifications in the past [65]. Early observations by Victor and colleagues [66] also suggest that a large proportion (21\%) of individuals with KS can make a full recovery. These clinical findings are consistent with current neuroimaging evidence that suggests the possibility of at least partial structural and functional recovery from alcohol-related brain damage if abstinence is maintained. A recent animal study reported that while the effects of chronic ethanol exposure (including working memory and episodic memory impairment) can recover with prolonged abstinence, the deficits of thiamine deficiency (spatial memory impairment and increased perseverative behavior) are more persistent [28].

\section{Clinical considerations}

The assessment and management of individuals with ARD and WKS entail a number of clinical issues. Firstly, a thorough nutritional and drinking history should be taken, with confirmation from an informant if possible. Secondly, all individuals with any evidence of chronic alcohol misuse and suspected of having WE should be treated immediately with parenteral thiamine [16]. Treatment with oral thiamine is ineffective because it does not achieve an adequate plasma concentration [67]. Furthermore, alcohol directly interferes with thiamine uptake [67]. While there is no consensus as to the optimum dose, frequency, route, and duration of thiamine treatment, the EFNS recommends that in cases of suspected WE, thiamine be given in doses of $200 \mathrm{mg}$ three times daily, preferably intravenously. This treatment should be continued until no further improvement in signs and symptoms is evident [19]. Thirdly, assessment of cognitive status should be conducted on an ongoing basis as this will allow any improvement, stabilization, or deterioration to be detected. Acute intoxication and withdrawal may exacerbate cognitive deficits, so assessment following this period (which usually lasts no longer than 2 weeks) may allow a more accurate baseline to be established. Fourthly, key characteristics associated with alcohol-related cognitive disorders may assist with differentiation from neurodegenerative conditions. These typically involve stabilization or improvement in cognition with abstinence; a cognitive profile involving executive, visuospatial, and memory difficulties with spared language function; and neurological symptoms such as ataxia. Neuroimaging may suggest atrophy in the mammillary bodies, thalamus and cerebellum, and ventricular enlargement, although this may vary from case to case.
Patients with ARD and WKS have shown cognitive improvement following treatment with memantine, although these findings require replication [68,69]. Finally, these socially isolated patients are often hospitalized for another health condition and this presents an ideal opportunity for screening, identification, and intervention.

\section{Conclusions}

The evidence reflects significant commonality between ARD and WKS. Neuropsychological studies have largely attempted to differentiate these syndromes by limiting individuals with more global cognitive impairment from WKS investigations and by excluding individuals with past symptoms of WKS from ARD studies. However, the validity of this distinction is now being brought into question. While attention has been given to the notion that ARD is likely related to underlying WKS pathology, it appears strange that more credence has not been given to whether WKS may clinically be a form of dementia, given that it typically entails multiple domains of cognitive impairment alongside functional deficits. While 'dementia' in current neurological settings is typically used to describe a progressive disease of the brain, it perhaps more accurately encompasses a deterioration of intellectual or cognitive function that may or may not be progressive in nature [70]. Debate over the utility of the term ARD in the clinical setting ensues [4]. It remains to be seen whether the term 'neurocognitive disorder' will satisfactorily resolve these nosological issues, dispel some of the stigma associated with this illness, and bring a better understanding of alcohol-related cognitive disorders.

\section{Abbreviations \\ ARBD, alcohol-related brain damage; ARD, alcohol-related dementia; DSM-IV, Diagnostic and Statistical Manual of Mental Disorders, 4th edition; EFNS, European Federation of Neurological Societies; KS, Korsakoff syndrome; WE, Wernicke's encephalopathy; WKS, Wernicke-Korsakoff syndrome.}

\section{Competing interests}

The authors declare that they have no competing interests.

\section{Author details}

'Dementia Collaborative Research Centre, School of Psychiatry, University of New South Wales, Anzac Parade, Kensington, NSW, 2052, Australia. 2Department of Psychology, Macquarie University, Balaclava Road, North Ryde, NSW, 2109, Australia. ${ }^{3}$ Academic Department for Old Age Psychiatry, Prince of Wales Hospital, Barker Street, Randwick, NSW, 2031, Australia. ${ }^{4}$ School of Public Health and Community Medicine, University of New South Wales, Anzac Parade, Kensington, NSW, 2052, Australia.

\section{Published: 25 January 2013}

\section{References}

1. Harper C: The neuropathology of alcohol-related brain damage. Alcohol Alcohol 2009, 44:136-140.

2. Blazer DG: The epidemiology of alcohol use disorders and subthreshold dependence in a middle-aged and elderly community sample. Am J Geriatr Psychiatry 2011, 19:685-694.

3. Plant M, Haw S: Women and Alcohol: Report of an Expert Seminar. Edinburgh: Health Education Board for Scotland and Health Research Centre; 2000.

4. Bowden SC: Alcohol-related dementia and Wernicke-Korsakoff syndrome. 
In Dementia. 4th edition. Edited by Ames D, Burns A, O'Brien J. London: Edward Arnold; 2010:730-737.

5. Moriyama Y, Mimura M, Kato M, Kashima H: Primary alcoholic dementia and alcohol-related dementia. Psychogeriatrics 2006, 6:114-118.

6. Gupta S, Warner J: Alcohol-related dementia: a 21st-century silent epidemic? Br J Psychiatry 2008, 193:351-353.

7. Jauhar S, Smith ID: Alcohol-related brain damage: not a silent epidemic BrJ Psychiatry 2009, 194:287-288

8. Goldstein G, Shelly C: Neuropsychological investigation of brain lesion localization in alcoholism. Adv Exp Med Biol 1980, 126:731-743.

9. Harper CM, Matsumoto I: Ethanol and brain damage. Curr Opin Pharmacol 2005, 5:73-78

10. Sullivan EV, Harding, AJ, Pentney R, Dlugos C, Martin PR, Parks MH, Desmond JE, Chen SHA, Pryor MR, De Rosa E, Pfefferbaum A: Disruption of frontocerebellar circuitry and function in alcoholism. Alcohol Clin Exp Res 2003, 27:301-309.

11. Bartsch AJ, Homola G, Biller A, Smith SM, Weijers HG, Wiesbeck GA, Jenkinson M, De Stefano N, Solymosi L, Bendszus M: Manifestations of early brain recovery associated with abstinence from alcoholism. Brain 2007 130:36-47.

12. Sullivan EV, Pfefferbaum A: Neurocircuitry in alcoholism: a substrate of disruption and repair. Psychopharmacology 2005, 180:583-594.

13. Bates ME, Barry D, Bowden SC: Neurocognitive impairment associated with alcohol use disorders: implications for treatment. Exp Clin Psychopharmacol 2002, 10:193-212.

14. Brust JC: Ethanol and cognition: indirect effects, neurotoxicity, and neuroprotection: a review. Int J Environ Res Public Health 2010, 7:1540-1557.

15. Crews FT, Collins MA, Dlugos C, Littleton J, Wilksons L, Neafsey EJ, Pentney R, Snell LD, Tabakoff B, Zou J, Noronha A: Alcohol-induced neurodegeneration: when, where and why? Alcohol Clin Exp Res 2004, 28:350-364.

16. Sechi GP, Serra A: Wernicke's encephalopathy: new clinical settings and recent advances in diagnosis and management. Lancet Neurol 2007, 6:442-455.

17. Sullivan EV, Pfefferbaum A: Neuroimaging of the Wernicke-Korsakoff Syndrome. Alcohol Alcohol 2009, 44:155-165.

18. Caine D, Halliday GM, Kril JJ, Harper CG: Operational criteria for the classification of chronic alcoholics: identification of Wernicke's encephalopathy. J Neurol Neurosurg Psychiatry 1997, 62:51-60.

19. Galvin R, Brathen G, Ivashynka A, Hillbom M, Tanasescu R, Leone MA: EFNS guidelines for diagnosis, therapy and prevention of Wernicke encephalopathy. Eur J Neuro 2010, 17:1408-1418.

20. Zahr NM, Kaufman KL, Harper CG: Clinical and pathological features of alcohol-related brain damage. Nat Rev Neurol 2011, 7:284-294.

21. Smith DM, Atkinson RM: Alcoholism and dementia. Int J Addict 1995, 30:1843-1869

22. Harper C: The incidence of Wernicke's encephalopathy in Australia a neuropathological study of 131 cases. J Neurol Neurosurg Psychiatry 1983, 46:593-598.

23. Torvik A, Lindboe CF, Rodge S: Brain lesions in alcoholics. J Neurol Sciences 1982, 56:233-248

24. Lishman WA: Alcoholic dementia: a hypothesis. Lancet 1986, 327:1 184-1186.

25. Akai J, Akai K: Neuropathological study of the nucleus basalis of meynert in alcoholic dementia. Arukoru Kenkyuto Yakubutsu Ison 1989, 24:80-88

26. Cullen K, Halliday G, Caine D, Kril J: The nucleus basalis (Ch4) in the alcoholic Wernicke-Korsakoff syndrome: reduced cell number in both amnesic and non-amnesic patients. J Neurol Neurosurg Psychiatry 1997, 63:315-320.

27. Vetreno RP, Hall JM, Savage LM: Alcohol-related amnesia and dementia: Animal models have revealed the contributions of different etiological factors on neuropathology, neurochemical dysfunction and cognitive impairment. Neurobiol Learn Mem 2011, 96:596-608.

28. Riberio AM, Pereira SR: Animal models of alcohol-induced dementia. In Neuromethods. Volume 48. Edited by De Deyn PP, Van Dam D. Springer; 2011:665-683. doi:10.1007/978-1-60761-898-0_33, @ Springer Science+Business Media, LLC 2011.

29. Homewood J, Bond NW: Thiamin deficiency and Korsakoff's syndrome: failure to find memory impairments following nonalcoholic Wernicke's encephalopathy. Alcohol 1999, 19:75-84.

30. Turner C: How much alcohol is in a 'standard drink'? An analysis of 125studies. Br J Addict 1990, 85:1171-1175.

31. Oslin DW, Streim JE, Parmelee P, Boyce AA, Katz IR: Alcohol abuse: a source of functional disability among residents of a VA nursing home. Int J Geriatr
Psychiatry 1997, 12:825-832

32. Horner MD, Waid LR, Johnson DE, Latham PK: The relationship of cognitive functioning to amount of recent and lifetime alcohol consumption in outpatient alcoholics. Addict Behav 1999, 24:449-453.

33. Parsons OA, Nixon SJ: Cognitive functioning in sober social drinkers: a review of the research since 1986. J Stud Alcohol Drugs 1998, 59:180-190.

34. Saxton J, Munro CA, Butters MA, Schramke C, McNeil MA: Alcohol, dementia, and Alzheimer's disease: comparison of neuropsychological profiles. $J$ Geriatr Psychiatry Neurol 2000, 13:141-149.

35. Oslin D, Atkinson RM, Smith DM, Hendrie H: Alcohol related dementia: proposed clinical criteria. Int J Geriatr Psychiatry 1998, 13:203-212.

36. Hulse GK, Lautenschlager NT, Tait RJ, Almeida OP: Dementia associated with alcohol and other drug use. Int Psychogeriatr 2005, 17 (Suppl):109-127.

37. Peters R, Peters J, Warner J, Beckett N, Bulpitt C: Alcohol, dementia and cognitive decline in the elderly: a systematic review. Age Aging 2008, 37:505-512.

38. Neafsey EJ, Collins MA: Moderate alcohol consumption and cognitive risk. Neuropsychiatric Disease Treatment 2011, 7:465-484.

39. Hoang T, Barnes D, Byers D, Byers A, Yaffe K: 20-year alcohol consumption patterns and cognitive impairment in older women. Alzheimer's Dementia 2012, 4 (Suppl):630.

40. Bate $C$, Williams A: Ethanol protects cultured neurons amyloid- $\beta$ and a-synuclein-induced synapse damage. Neuropharmacology 2011, 61:1406-1412.

41. American Psychiatric Association: Diagnostic and Statistical Manual of Mental Disorders. 4th edition. Washington, DC: American Psychiatric Association; 1994.

42. Chung YA, Choi SW, Joe KH: Regional cerebral blood flow in patients with alcohol-related dementia: a SPECT study. Int J Neurosci 2009, 119:2100-2111.

43. Oslin DW, Cary MS: Alcohol-related dementia: validation of diagnostic criteria. Am J Geriatr Psychiatry 2003, 11:441-447.

44. Schmidt KS, Gallo JL, Ferri C, Giovannetti T, Sestito N, Libon DJ, Schmidt PS: The neuropsychological profile of alcohol-related dementia suggests cortical and subcortical pathology. Dement Geriatr Cogn Disord 2005, 20:286-291.

45. World Health Organisation: ICD-10 Classifications of Mental and Behavioural Disorder: Clinical Descriptions and Diagnostic Guidelines. Geneva: World Health Organisation; 1992

46. American Psychiatric Association: DSM-5 development: proposed revisions [www.dsm5.org/proposedrevision].

47. Ritchie K, Villebrun D. Epidemiology of alcohol-related dementia. Hand Clin Neurol 2008, 89:845-850.

48. Carlen PL, McAndrews MP, Weiss RT, Dongier M, Hill JM, Menzano E, Farcnik K, Abarbanel J, Eastwood MR: Alcohol-related dementia in the institutionalized elderly. Alcohol Clin Exp Res 1994, 18:1330-1334.

49. Bello VM, Schultz RR: Prevalence of treatable and reversible dementias: a study in a dementia outpatient. Dement Neuropsychol 2011, 5:44-47.

50. McMurtray A, Clark DG, Christine D, Mendez MF: Early-onset dementia: frequency and causes compared to late-onset dementia. Dement Geriatr Cogn Disord 2006, 21:59-64.

51. Harvey RJ, Skelton-Robinson M, Rossor MN: The prevalence and causes of dementia in people under the age of 65 years. I Neurol Neurosurg Psychiatry 2003, 74:1206-1209.

52. Draper B, Karmel R, Gibson D, Peut A, Anderson P: Alcohol-related cognitive impairment in New South Wales hospital patients aged 50 years and over. Aust N Z J Psychiatry 2011, 45:985-992.

53. Lennane KJ: Patients with alcohol-related brain damage: therapy and outcome. Aust Drug Alcohol Rev 1988, 7:89-92.

54. Boden JM, Fergusson DM: Alcohol and depression. Addiction 2011, 106:906-914

55. Blansjaar BA, Horjus MC, Nijhuis HGJ: Prevalence of the Korsakoff syndrome in The Hague, The Netherlands. Acta Psychiatr Scand 1987, 75:604-607.

56. Ramayya A, Jauhar P: Increasing incidence of Korsakoff's psychosis in the east end of Glasgow. Alcohol Alcohol 1997, 32:281-285.

57. Munro CA, Saxton J, Butters MA: Alcohol dementia: 'cortical' or 'subcortical' dementia. Arch Clin Neuropsychol 2001, 16:523-533.

58. Fama R, Marsh L, Sullivan EV: Dissociation of remote and anterograde memory impairment and neural correlates in alcoholic Korsakoff syndrome. J Int Neuropsychol Soc 2004, 10:427-441.

59. Kopelman MD, Thomson AD, Guerrini I, Marshall EJ: The Korsakoff syndrome: 
clinical aspects, psychology and treatment. Alcohol Alcohol 2009, 44:148-154.

60. Van Oort R, Kessels RP: Executive dysfunction in Korsakoff's syndrome: time to revise DSM criteria for alcohol-induced persisting amnestic disorder? Int J Psychiatry Clin Prac 2009, 13:78-81.

61. Dirksen CL, Howard JA, Cronin-Golomb A, Oscar-Berman M: Patterns of prefrontal dysfunction in alcoholics with and without Korsakoff's syndrome, patients with Parkinson's disease, and patients with rupture and repair of the anterior communicating artery. Neuropsychiatr Dis Treat 2006, 2:327-339

62. Cutting J: The relationship between Korsakov's syndrome and 'alcoholic dementia'. BrJ Psychiatry 1978, 132:240-251.

63. Scheurich A: Neuropsychological functioning and alcohol dependence. Curr Opin Psychiatry 2005, 18:319-323.

64. Woodburn KJ: Early-onset dementia in Lothian, Scotland: an analysis of clinical features and patterns of decline. Health Bull 1999, 57:384-392.

65. Fujiwara E, Brand M, Borsutzky S, Steingass HP, Markowitsch HJ: Cognitive performance of detoxified alcoholic Korsakoff syndrome patients remains stable over two years. J Clin Exp Neuropsychol 2008, 30:576-587.

66. Victor M, Adams RD, Collins GH: The Wernicke-Korsakoff syndrome. A clinical and pathological study of 245 patients, 82 with post-mortem examinations. Contemp Neurol Ser 1971, 7:1-206.
67. Thomson AD, Marshall EJ: The treatment of patients at risk of developing Wernicke's Encephalopathy in the community. Alcohol Alcohol 2006, 41:159-167.

68. Cheon Y, Park J, Joe KH, Kim DJ: The effect of 12-week open-label memantine treatment on cognitive function improvement in patients with alcohol-related dementia. Int J Neuropsychopharmacol 2008, 11:971-983.

69. Rustembegovic A, Kundurovic Z, Sapcanin A, Sofic E: A placebo-controlled study of memantine (Ebixa) in dementia of Wernicke-Korsakoff syndrome. Med Arkhiv 2003, 57:149-150.

70. Ropper AH, Samuels MA: Dementia and the amnesic (Korsakoff) syndrome with comments on the neurology of intelligence and memory. In Adams and Victor's Principles of Neurology. 9th edition. Edited by Ropper AH, Samuels MA. New York: The McGraw-Hill Companies; 2009:367-383.

doi:10.1186/alzrt157

Cite this article as: Ridley NJ, et al: Alcohol-related dementia: an update of the evidence. Alzheimer's Research \& Therapy 2013, 5:3. 